\title{
Editorial
}

\section{eCAM: Retaining an International Perspective}

\section{Edwin L. Cooper}

Laboratory of Comparative Neuroimmunology, Department of Neurobiology, David Geffen School of Medicine at UCLA, University of California, Los Angeles, CA 90095-1763, USA

Corresponding author: Edwin L. Cooper, Laboratory of Comparative Neuroimmunology, Department of Neurobiology, David Geffen School of Medicine at UCLA, University of California, Los Angeles, CA 90095-1763, USA. Tel: (310) 825-9567; Fax: (310) 825-2224; E-mail: ecam@mednet.ucla.edu

eCAM seemed to have arrived at the right time, 2004, when there was a growing need for populations to understand the biological basis of health and to take charge. Before the pharmaceutical industry dawned, humans did for themselves as best they could. Their innovations were limitless despite the sparse or even non-existent biotech instruments and MD's prescriptions. Yet their potions, incantations and anecdotes did for them what modern university medical centers and the pharmaceuticals and supplements now do. Still the sages of ancient medicine remain but their age-old clout is meshing with more open views by Western practitioners. The meeting is still not yet at the halfway mark but lines are blurring.

As science became increasingly intertwined with medicine, there emerged greater and more numerous discoveries that were tried and tested and offered as cures or perhaps 'managers' that would in many instances replace the shaman and certain folk remedies. Many of them were passed on generation after generation verbally and surely not published in evidence-based biomedical journals. Yet, they have not been entirely abandoned and now provide a vast array of seeds that are being strewn on to the most fertile soil rich with nutritious advancements in the form of highly sophisticated instruments and defined formulas designed for and that insure healthier and even longer lives.

For older cultures, primarily in Asia, had no scientific biomedical journals in which to document evidence that is largely now dominated by the West. The emergence of the new biomedicine began to monopolize the world and in many instances replaced the prevailing healing powers and concoctions of ancient cultures. Knowing these constraints hopefully reasoned formulations and fast-forward, $e C A M$ began with an international flourish and continues to this day. As a last tribute, I review a select group of pertinent papers that will be our last in volume 7 which has been supported most recently and entirely by OUP.

Professors Seung-Hoon Choi and Il-Moo Chang of the College of Oriental Medicine, Kyung Hee University and Natural Products Research Institute have indeed done a credible international service. They have assisted the WHO to publish a dictionary-type book entitled 'WHO International Standard Terminologies on Traditional Medicine in the Western Pacific Region'. It has a total of 3259 technical terms commonly used in traditional Chinese (TCM), Japanese (Kampo) and Vietnamese medicines. This comprehensive guide includes the English expression, the original Chinese character and a concise English definition. There are 3106 terms from basic theories, diagnostics, diseases and therapeutics, which include acupuncture and moxibustion and even the English wording of 153 titles which are considered the most important traditional medical classics published in these four countries. There is a prominent feature, the codification format that assigns numbers in hundred decimal units for each category. This ensures flexibility for adding more future terminologies. This volume is also useful in constructing a database for retrieving various published scientific articles. Using these standard terminologies is certainly advantageous to deliver accurate meanings and ultimately to avoid many expressions for a single term in different scientific manuscripts of Oriental medicine.

Moving now closer to the Mediterranean and the Middle East, relatively new terminologies have emerged. Azaizeh et al remind us of several points. Complementary medicine is a formal method of health care in most 
countries of the ancient world. It is expected to become more widely integrated into the modern medical system, including the medical curriculum. As an example, herbal medicine can be broadly classified into four basic systems as follows: Traditional Chinese Herbalism, Ayurvedic Herbalism, Western Herbalism-which originally came from Greece and Rome to Europe and then spread to North and South America - and Traditional Arabic and Islamic Medicine (TAIM). Clearly the concept of Arabic traditional herbal medicine is another component of modern life in the Middle East, and it is acquiring worldwide respect, with growing interest among traditional herbalists and the scientific community. TAIM therapies reveal remarkable success in healing acute and chronic diseases and have been utilized by people in the Mediterranean who have faith in spiritual healers. TAIM is the first choice for many in dealing with infertility, epilepsy, psychosomatic troubles and depression. Moreover, efficacy and safety of complementary medicine have become increasingly important and supervision of the techniques and procedures is required for commercial as well as traditional uses. More research is therefore needed to understand this type of medicine and to ensure its safe usage. In partnership with TAIM there are the usual Western medical approaches.

Moving now to a somewhat distant but related subject, let us consider a clinical approach. Bertrand Graz reminds us that the ancient Greek medical theory based on balance or imbalance of humors disappeared in the Western world. Since it still survives elsewhere, we pose the question is this survival related to a certain degree of health care efficiency? This hypothesis was tested through Graz's study of classical Greco-Arab medicine in Mauritania. Here is a brief review of the conditions modern general practitioners evaluated with respect to the safety and effectiveness of classical Arabic medicine in a Mauritanian traditional clinic. This was accompanied by a prognosis/follow-up method that allows the following comparisons: (i) actual patient progress (clinical outcome) compared with what the traditional 'tabib' had anticipated (prognostic ability); and (ii) patient progress compared with what could be hoped for if patients were treated by a modern Western physician in the same neighborhood. According to Graz, practice appeared fairly safe and, on average, the clinical outcome was similar to what could be expected after using modern medicine so that in some cases, patient progress was 'better' than expected. So what are the implications? Prognosis and outcome studies such as this one may help to develop institutions where: patients find support in making their choices, among several treatment options and medical systems. Both choices are indeed implicit in the practice of CAM.

Finally, back to Japan and a CAM modality of international importance. Acupuncture originated in China and is widespread throughout Asia and substantial movement outside. Still there is a higher utilization when compared to Western countries. Ishizaki and a team in Kyoto conducted annual nationwide surveys from 2003 to 2006 to understand the utilization of acupuncture in Japan. Face-to-face interviews were conducted with 2000 individuals randomly chosen from the resident database. Reasons given for continuing therapy included the effective amelioration of symptoms, comfort of the procedure and low number of side-effects, while those who decided against continuing cited no improvement of symptoms, cost and lack of time for treatment. Application of the treatment for musculoskeletal problems and utilization by the older population were specific reasons for prominent users of acupuncture and/or moxibustion in Japan.

These brief reviews have highlighted the international thrust of publications in eCAM. With the last issue, volume 7, no 3, you saw how approaches: hypotheses, basic science and clinical analyses can be amalgamated to attack a single problem, now of epidemic proportions and one that is partially self inflicted and not caused by parasites or a poor environment. Now eCAM is moving on, flying away from OUP its birth nest, perhaps no more a fledging but now easing toward an approaching adolescence. Leaving OUP is bittersweet. My friendship will endure with Prof. Nobuo Yamagachi and Patty Willis and I owe a lot to the generosity of Martin Richardson and Charlotte Brabants and Akiko Tanaka. I have also received sympathetic and highly cooperative support from our $e C A M$ Editorial Board.

As a contemporary extension of ancient practices, maybe there is a hidden message in our becoming allied with Hindawi Publishing Corporation in Egypt, a country whose culture may be arguably older than India and China the birthplaces of TCM and Ayurveda. I will continue as Editor in Chief with a wind of renewed vigor not only for pleasure of continued nurturing of $e C A M$; there is more. No doubt I will have a chance to visit Cairo and its institutions. I spent my third sabbatical year at Cairo University, (in 1985-86) Department of Zoology. Some of my colleagues are still around along with others at the American University Cairo, the National Research Center at Dokki and the NAMRU Onword!

\section{References}

1. Choi SH, Chang IM. A milestone in codifying the wisdom of traditional Oriental Medicine: TCM, Kampo, TKM, TVM-WHO international standard terminologies on traditional medicine in the Western pacific region. eCAM January 5, 2009; doi: 10.1093/ ecam/nen083 [Epub ahead of print].

2. Azaiszeh H, Saad B, Cooper EL, Said O. Traditional Arabic and Islamic Medicine, a re-emerging health aid. eCAM June 13, 2008; doi: 10.1093/ecam/nen039 [Epub ahead of print].

3. Graz B. Prognostic ability of practitioners of traditional Arabic Medicine: comparison with Western methods through a relative patient progress scale. eCAM March 13, 2008; doi: 10.1093/ecam/ nen022 [Epub ahead of print].

4. Ishizaki N, Yano T, Kawakita K. Public status and prevalence of Acupuncture in Japan. eCAM June 19, 2008; doi: 10.1093/ecam/ nen037 [Epub ahead of print]. 


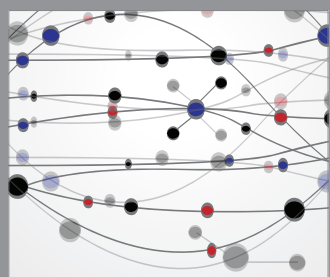

The Scientific World Journal
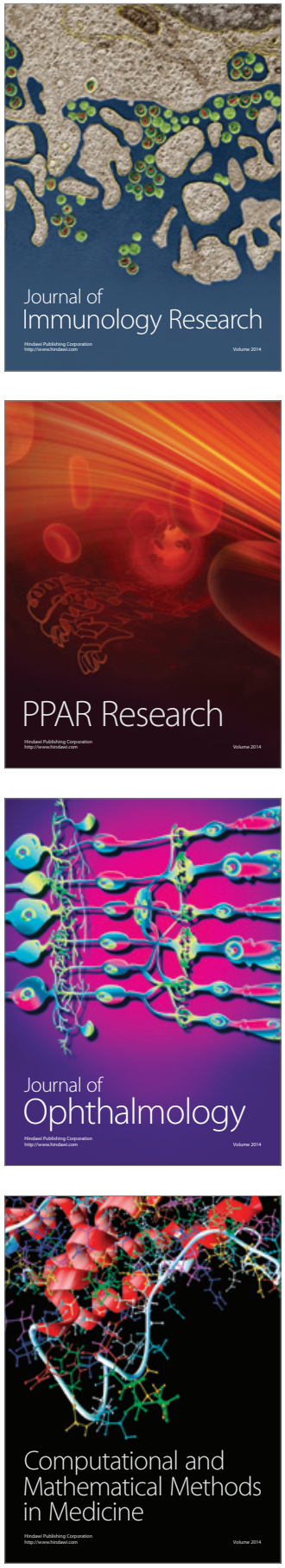

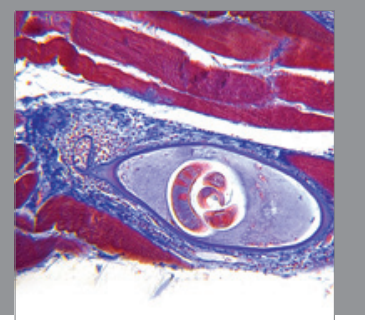

Gastroenterology

Research and Practice
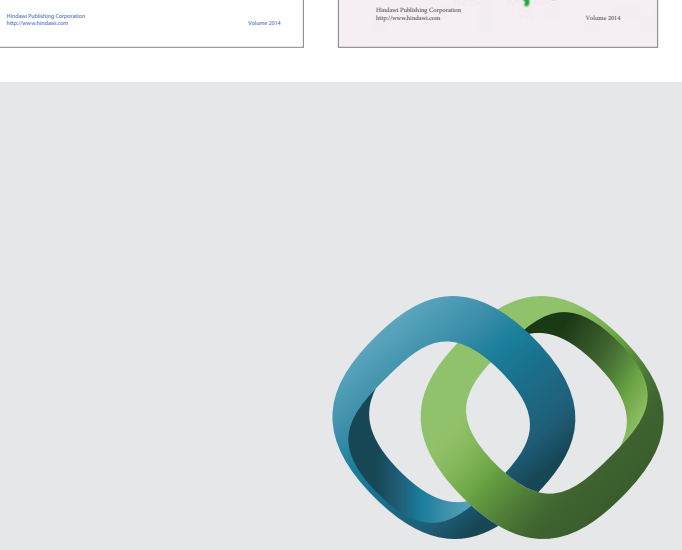

\section{Hindawi}

Submit your manuscripts at

http://www.hindawi.com
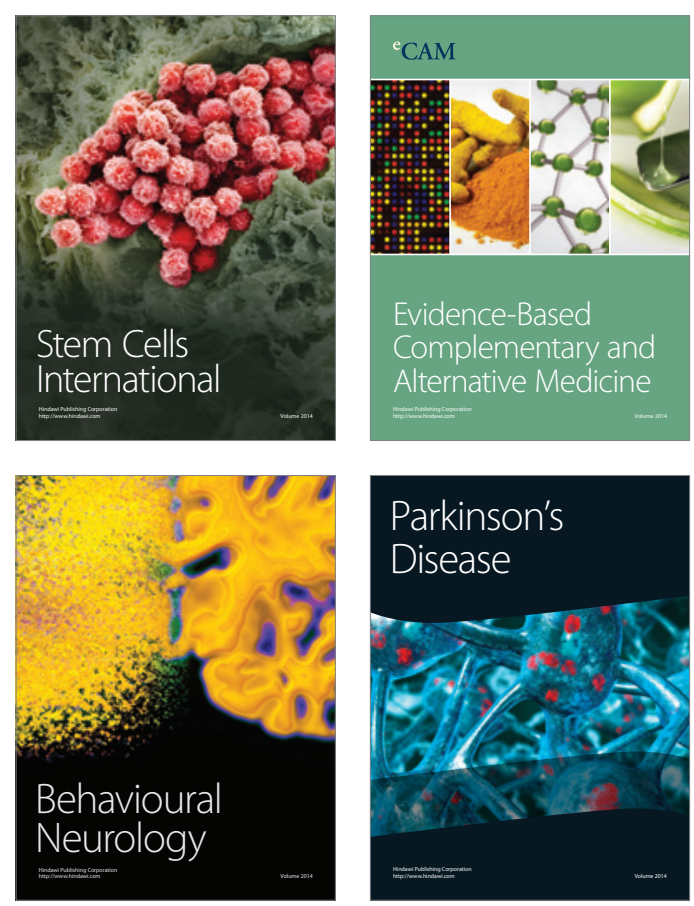

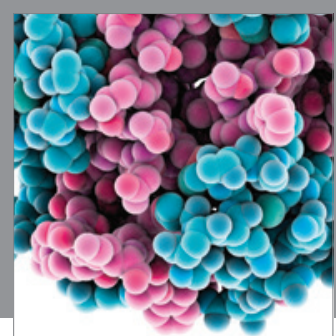

Journal of
Diabetes Research

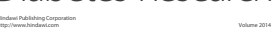

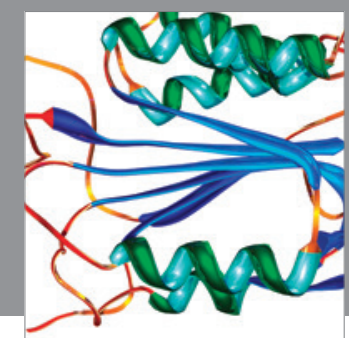

Disease Markers
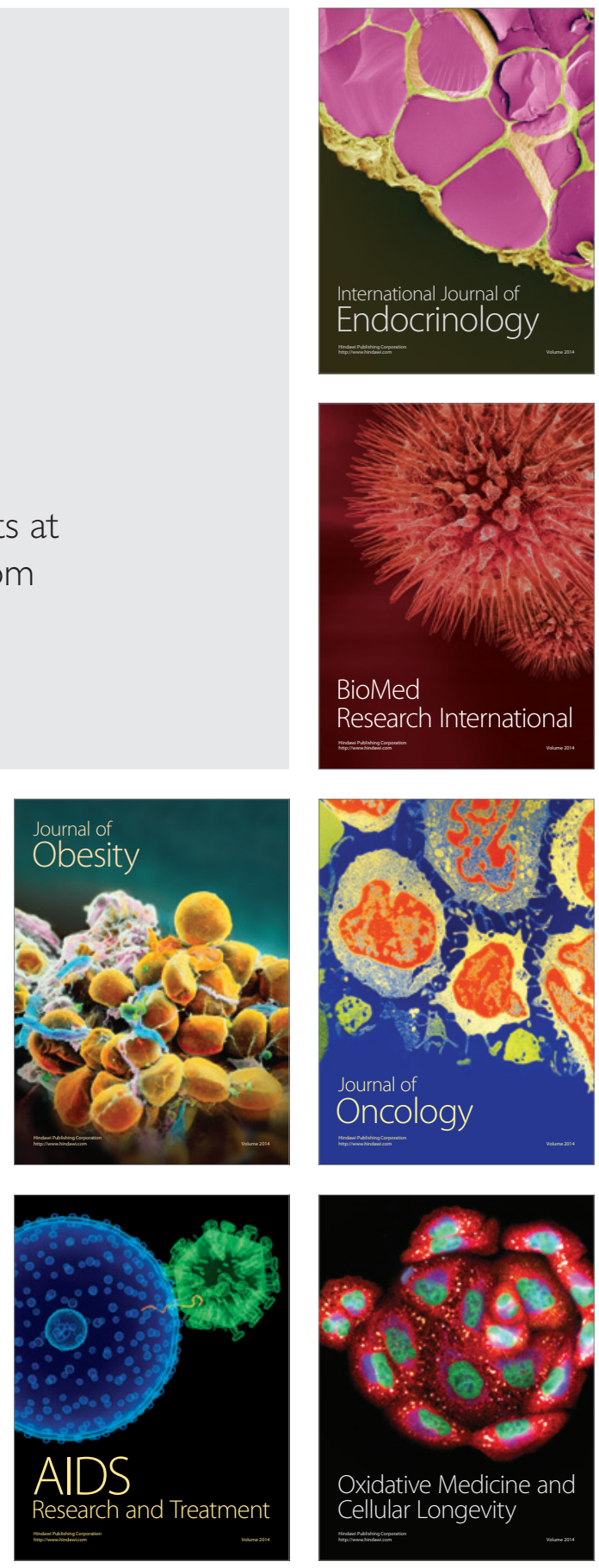